\title{
Application of Artificial Neural Network to Predict Water Levels in Virginia Key, Florida
}

\author{
W. Huang \\ Department Hydraulic Engineering, Tongji University, \\ Shanghai, China \\ Department of Civil Engineering, Florida State University, \\ Florida, U.S.A.
}

\author{
S.D. $\mathrm{Xu}$ \\ Department of Harbor, Waterway and Coastal Engineering \\ School of Transportation, Southeast University \\ Nanjing, China
}

\author{
Y.N. Chao \\ Department Hydraulic Engineering, Tongji University \\ Shanghai, China
}

\begin{abstract}
This paper presents the application of the artificial neural network to predict long-term water level in Virginia Key, south Florida. Model input is based on the NOAA observed data at a remote station, Cedar Key station located at about $584 \mathbf{~ k m}$ away. Results indicate that, even though the long distance between two stations, neural network model predictions of water levels are satisfactory, with a $\mathbf{0 . 8 6}$ correlation coefficient. Model accuracy may be further improved by adding more factors, such wind speed and direction, in future studies.
\end{abstract}

Keywords-artificial neural network; coastal water levels; Cedar Key; Virginia Key; Florida

\section{INTRODUCTION}

Water level data collection is an important part in supporting NOAA's mission. Long-term water level data can be very helpful for researchers to analyze the patterns of tidal waves and hurricane storm surges in coastal areas. Data are also required in calibrate and verify hurricane storm surge models for surge forecasting. In the coastal water of Florida, there are a number of long-term water level observation stations operated by NOAA. However, the numbers of water level stations are far from enough to provide sufficient details of resolution to variations of water levels and hurricane surges. More data for locations between NOAA stations at finer resolution are needed. More data at locations at inland waters, such as estuaries and bays and coastal water ways, are needed. Increasing the number and quality of long-term water level data is of importance for hurricane research. For example, in order to determine the storm surge hydrograph with 50 year return interval peak elevation, over 50 years of water level data may be needed. However, for many water lever observations conducted by some state agencies, only short term data are available, mostly ranging from a few weeks to a few years. Without long-term historic data, many agencies have to roughly estimate 50 year or 100 year storm surge hydrographs generated from the computational hydrodynamic model.

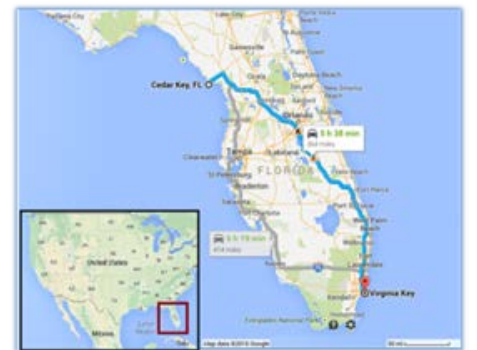

FIGURE I . LOCATIONS CEDAR KEY STATION AND VIRGINIA KEY STATION IN THE STUDY AREA OF SOUTH FLORIDA, U.S.A.

Artificial neural networks (ANN) have been widely used in real-time control modeling and multivariate non-linear time series modeling in the many research areas such as electronics, aerospace, and manufacturing engineering. An ANN can correlate multiple input variables with the output signal [1] through nodes or neurons. It is capable of directly correlating input time series of forcing functions to the output variables through the interconnected nodes with trainable weights and bias. In fact, the neural network is a recognized powerful tool which has been widely used in multivariate signal processing and control in the areas such as electronics, aerospace, and manufacturing engineering. In contrast to the traditional circulation modeling, the neural network model is trained and validated from data. A neural network model does not require data of bottom roughness, bathymetry and topography, and dispersion and eddy viscosity. However, it can provide information only at locations where input data are available for model training. In applying a trained and validated ANN, output variables are directly calculated without iteration from the input variables and the vectors of weights and bias in the network nodes. This functioning is similar to directly find the output from a linear regression function. Therefore, applying an ANN model takes much less computational time than the traditional fluid mechanic models, if data are available to establish the ANN model.

In this study, a neural network model was tested for predicting water level in Virginia Key in Atlanta Coast near Miami based on the observed water level data in Cedar Key in the Gulf Coast, Florida. The distance between those two 
stations are approximately 363 miles (584 km). Long-term data (almost 90 years) are available in NOAA station in Cedar Key. The successfully development of the ANN model between Cedar Key and Virginia Key will provide a low-cost tool to increase the number of water level and storm surge data for hurricane storm surge research in the coast of Miami.

\section{ARTIFICIAL NEURAL NETWORK DESCRIPTION}

In analogy to the biological nervous system, ANN technology is being applied to solve a wide variety of complex scientific, engineering and business problems. Neural networks are ideally suited for such problems because like their biological counterparts, an ANN can learn, and therefore be trained to find solutions, recognize patterns, classify data, and forecast future events. Hagan and Demuth [1] and Haykin [2] give the details of the theories and engineering applications of the neural networks. In a neural network model, the outputs are correlated to the inputs through the neurons (or nodes) with weights and bias. The behavior of a neural network is defined by the way its individual computing elements are connected and by the strength of those connections or weights. The weights are automatically adjusted by training the network according to a specific learning rule until it performs with the desired error rate.

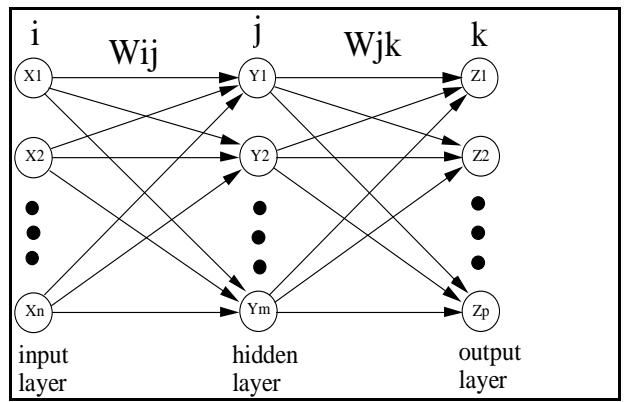

FIGURE II . A THREE-LAYER FEED-FORWARD NEURAL NETWORK FOR MULTIVARIATE SIGNAL PROCESSING.

\section{A. Multiple-Layer Model}

In practical applications, a neural network often consists of several neurons in several layers. A schematic diagram of a three-layer neural network is given in Figure II, where $X_{i}$ $(\mathrm{i}=1, \ldots, n)$ represents the input variables (such as boundary forcing functions of wind and water levels); $Y_{i}(i=1, \ldots, m)$ represents the outputs of neurons in the hidden layer; and $\mathrm{Z}_{\mathrm{i}}$ $(\mathrm{i}=1, \ldots, p)$ represents the outputs of the neural network such as water levels and currents in and around coastal inlets. The layer that produces the network output is called the output layer, while all other layers are called hidden layer. The weight matrix connected to the inputs is called the input weight $\left(\mathbf{W}_{\mathbf{i j}}\right)$ matrix, while the weight matrices coming from layer outputs are called layer weights $\left(\mathbf{W}_{\mathbf{j k}}\right)$.

\section{B. Standard Network Training Using Gradient Descent Method}

Multiple-layer neural network using back propagation training algorithm is popular in neural network modeling [1] because of its ability to recognize the pattern and relationship between non-linear signals. The term of back propagation usually refers to the manner in which the gradients of weights are computed for non-linear multi-layer networks. A neural network must be trained to determine the values of the weights that will produce the correct outputs. Mathematically, the training process is similar to approximate a multi-variable function, $\boldsymbol{g}(\boldsymbol{X})$, by another function of $\boldsymbol{G}(\boldsymbol{W}, \boldsymbol{X})$, where $X=\left[x_{1}, x_{2}, \ldots, x_{n}\right]$ is the input vector, and $W=\left[w_{1}, w_{2}, \ldots . w_{n}\right]$ the coefficient or weight vector. Therefore, the training task is to find the weight vector ' $W$ ' that provides the best approximation of the function $g(X)$ based on the training input $[X]$.

The standard or basic training method is called 'Gradient Descent Method'; in which weight changes move the weights in the direction where the error declines most quickly. Training is carried out by assigning random initial weights to each of the neurons (usually between 0.1 to 1.0), and then presenting sets of known input and target (output) values to the network. The network estimates the output value from the inputs, compares the model predicted output to the target value, and then adjusts the weights in order to reduce the mean squared difference between the network output and the target values. The complete input-output sets are often run through the network for several iterations (or epochs) until either the mean square error is reduced to a given level or reaches a minimum, or until the network has been trained for a given number of iterations.

If we let $w_{m}$ represent the value of weight $w$ after $m$-th iteration in a neuron, then

$$
w_{m}=w_{m-1}+\Delta w_{m}
$$

Where $\Delta \mathrm{w}_{\mathrm{m}}$ is the change in the weight $w$ at the end of iteration $m$. It is calculated by

$$
\Delta w_{m}=-\varepsilon d_{m}
$$

Where $\varepsilon$ is the user-specified parameter controlling the proportion by which the weights are modified. The term $d_{m}$ is given by

$$
d_{m}=\sum_{n=1}^{n}\left(\frac{\partial E}{\partial w_{m}}\right)
$$

Where $N$ is the total number of examples, and $E$ is the simulation output error.

\section{Network Development Processes}

In neural network model development, the first step is to design a specific network architecture (that includes a specific number of "layers" each consisting of a certain number of "neurons"). The size and structure of the network needs to match the nature (e.g., the formal complexity) of the investigated phenomenon. Because the latter is obviously not known well at this early stage, this task is not easy and often involves multiple "trials and errors." (Now, there is, however, neural network software that applies artificial intelligence techniques to aid in that tedious task and finds "the best" network architecture.) The new network is then subjected to the process of "training." In that phase, neurons apply an iterative process to the number of inputs (variables) to adjust 
the weights of the network in order to optimally predict (in traditional terms one could say, find a fit to) the sample data on which the training is performed. After the phase of learning from an existing data set, the new network is ready and it can then be used to generate predictions.

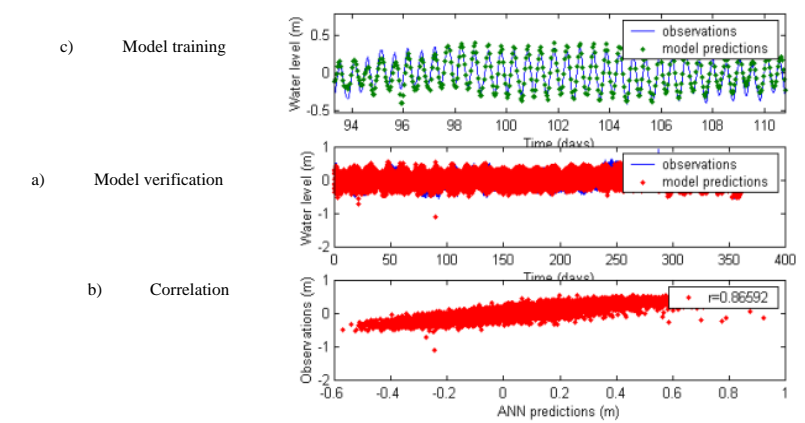

FIGURE III. NEURAL NETWORK MODEL TRAINING (A) AND VERIFICATION (B), AND CORRELATION (C) BETWEEN MODEL PREDICTIONS AND OBSERVATIONS OF WATER LEVELS DURING THE VERIFICATION PERIOD.

\section{ApPlication ANN to COASTAL PREDict COASTAL WATER LEVELS}

The ANN model was developed under Matlab software environment as described by Huang an Foo [3] and Huang et al. [4]. Two years of data at Cedar Key and Virginia Key (1999 and 2002) were elected to train and verify the ANN model. The first year (1999) data were used in model training, and the $2^{\text {nd }}$ year data were used in model verification. As shown in Figure 3, model predictions math well with observations. Results indicate that the ANN model provide reasonable predictions of water levels at Virginia Key station, with the correlation coefficient of 0.866 . Considering that the distance between Cedar Key and Virginia Key is very long, approximately $584 \mathrm{~km}$, the results are encouraging. The accuracy can be further improved in future study by adding additional factors that may affect water levels, such as wind speed and directions. Because NOAA maintains long-term water level data observations (over 90 years) at Cedar Key station, the ANN model developed in this study would make it possible to predict long-term water levels at Virginia Key. The data would be very helpful to support coastal hazards analysis for the city of Miami, which is located near Virginia Key.

\section{CONCLUSION}

In this study, a neural network model has been applied to predict coastal water levels at Virginia Key station, near Miami, Florida, U.S.A. Water level observations at Cedar Key station, which is located at about $584 \mathrm{~km}$ away, was used as the input. Data in 1999 were used in model training, and data in 2002 were used in model verification. Despite the long distance between these stations, model predictions of water levels reasonably match the observations, with a correlation coefficient of 0.866 . The model can be further improved by adding wind factors in the future study. The data at Virginia Key derived from the ANN model will be helpful to support coastal hazard study.

\section{REFERENCES}

[1] Hagan, M.T., Demuth, H., and Beale, M., Neural Network Design, PWS Publishing Company, 20 Park Plaza, Boston, MA 02116 - 4324, 1995.

[2] Haykin, S., Neural Network: A Compressive Foundation. Prentice Hall, New Jersey, 1999.

[3] Huang, W. \& Foo, S., Neural network modeling of salinity variation responding to multiple forcing functions in Apalachicola River, Water Research, vol. 36, pp. 356-362, 2002.

[4] Huang, W., Murray, C., Kraus, N. \& Rosati, J., Development of a regional neural network for coastal water level predictions, Ocean Engineering, vol. 30, pp. 2275-2295, 2003. 